\title{
Angular distributions and critical minima in the elastic scattering of electrons by atomic Copper
}

\author{
Shorifuddoza M. ${ }^{1,1}$, Pretam Das ${ }^{1}$, Raihan Kabir ${ }^{2}$, A. K. Haque ${ }^{2}$, and M Uddin ${ }^{1}$ \\ ${ }^{1}$ Pabna University of Science and Technology \\ ${ }^{2}$ University of Rajshahi
}

May 11, 2020

\begin{abstract}
Dirac relativistic partial wave analysis has been employed to analyze the angular distributions and critical minima along with maximum spin polarization for the elastic scattering of electrons from copper atoms over the energy range $1-2000 \mathrm{eV}$. Integrated elastic, inelastic, total and momentum transfer cross sections have also been calculated. This work uses a complex electronatom optical potential that includes static, exchange, correlation-polarization and absorption potentials. Comparison of our calculations with the available experimental data and other theoretical calculations show a satisfactory agreement. As far as we are concern, critical minima and corresponding maximum spin polarization points have not yet been reported in literature.
\end{abstract}

\section{Hosted file}

$\mathrm{Cu}$ Manuscript.pdf available at https://authorea.com/users/313439/articles/449948-angulardistributions-and-critical-minima-in-the-elastic-scattering-of-electrons-by-atomiccopper

\section{Hosted file}

$\mathrm{Cu}$ Manuscript.tex available at https://authorea.com/users/313439/articles/449948-angulardistributions-and-critical-minima-in-the-elastic-scattering-of-electrons-by-atomiccopper 
figures/F1-DCS/F1-DCS-eps-converted-to.pdf 
figures/F2-DCS/F2-DCS-eps-converted-to.pdf 
figures/F3-DCS/F3-DCS-eps-converted-to.pdf 
figures/F4-CM/F4-CM-eps-converted-to.pdf 
figures/F6-CM3/F6-CM3-eps-converted-to.pdf 
figures/F5a-3D-DCS/F5a-3D-DCS-eps-converted-to.pdf 
figures/F5b-3D-S/F5b-3D-S-eps-converted-to.pdf 
figures/F6-TCS/F6-TCS-eps-converted-to.pdf 